\title{
Klotho expression is correlated to molecules associated with epithelial-mesenchymal transition in lung squamous cell carcinoma
}

\author{
TAKAYUKI IBI, JITSUO USUDA, TATSUYA INOUE, AKIRA SATO and KYOSHIRO TAKEGAHARA \\ Department of Thoracic surgery, Nippon Medical School, Tokyo 113-8603, Japan
}

Received August 27, 2016; Accepted March 7, 2017

DOI: $10.3892 / \mathrm{ol} .2017 .6862$

\begin{abstract}
Klotho is known as an anti-aging gene. We previously reported that the expression of Klotho is a postoperative prognostic factor for patients with lung large cell neuroendocrine carcinoma and lung small cell carcinoma. Recently, Klotho was shown to suppress the epithelial-mesenchymal transition (EMT). In the present study, we examined the association between the expression of Klotho and the regulation of EMT in lung squamous cell carcinoma. We immunohistochemically examined the expression of Klotho in patients with lung squamous cell carcinoma who had undergone surgical resection or photodynamic therapy. The immunohistochemical analysis showed that Klotho expression was observed not only in normal bronchial epithelial cells, but also in centrally located early lung cancers, which were all carcinomas in situ and were treated using PDT. However, in lung cancer patients with invasive and or advanced squamous cell carcinoma who had undergone a complete surgical resection, Klotho expression was observed in only 4 patients $(13 \%)$. To elucidate the associations between the expression of Klotho and the expressions of EMT-related proteins, such as E-cadherin, N-cadherin, vimentin, and Snail, we transiently transfected GFP-Klotho plasmid DNA into the human squamous lung cancer cell line SQ5 and examined the expressions of these proteins of GFP-positive cells after sorting using flow cytometry. In SQ5 cells overexpressing GFP-Klotho, the expression of $\mathrm{N}$-cadherin, which is a mesenchymal marker, was completely inhibited, compared with that in SQ5 cells transfected with the GFP vector. The overexpression of Klotho did not affect the regulation of either other mesenchymal markers (such as vimentin and Snail) or the regulation of an epithelial marker (E-cadherin). We concluded that the expression of Klotho was
\end{abstract}

Correspondence to: Dr Jitsuo Usuda, Department of Thoracic Surgery, Nippon Medical School, 1-1-5 Sendagi, Bunkyo, Tokyo 113-8603, Japan

E-mail: jusuda@nms.ac.jp

Key words: Klotho, epithelial-mesenchymal transition, lung cancer and squamous cell carcinoma related to the degree of cancer invasiveness and that Klotho inhibits the expression of N-cadherin and regulates the EMT in lung cancer.

\section{Introduction}

Lung cancer is a relatively common cancer and is a major cause of cancer-related death worldwide $(1,2)$. The majority of lung cancers are non-small cell cancers (NSCLC), consisting of adenocarcinomas and squamous cell carcinomas. Treatment for NSCLC has improved dramatically in the past 15 years thanks to molecular-targeted drugs (3-5). Recently, new molecular-targeted drugs or immunotherapy drugs, such as immune checkpoint inhibitors, have been developed, and treatment strategies, particularly those for squamous cell carcinoma, have been improved using personalized medicine $(6,7)$. However, the outcome of cancer treatment is still far from satisfactory, and further examinations are required.

The epithelial-mesenchymal transition (EMT) is a biological step during which epithelial cells lose their cell polarity and cell-cell adhesion. This change is considered an important step in both invasion and metastasis and the development of treatment resistance in cancer. Cancer cells that have undergone an EMT acquire the ability to infiltrate and migrate to other organs $(8,9)$. Recent studies have suggested that the EMT is also involved in the acquisition of characteristics of cancer-stem-like cells $(10,11)$. Therefore, we aimed to analyze the EMT-related mechanism of squamous cell carcinoma and to develop a new treatment strategy for squamous cell carcinoma of the lung.

The Klotho gene, which is a 1014-amino acid single-pass transmembrane protein expressed predominantly in renal tubular epithelial cells, has been characterized as a systemic anti-aging hormone and was originally identified in mice homozygous for the mutated allele $\left(\mathrm{kl}^{-/}\right)$(12-14). These mice show a human-like aging-related syndrome and develop multiple disorders such as hypogonadism, ectopic calcification, osteoporosis, skin atrophy, and pulmonary emphysema (13). In contrast, transgenic mice overexpressing Klotho show an extended life span that is $30 \%$ longer in males and $20 \%$ longer in females. Previously, we reported an association between the level of Klotho expression and overall survival in patients with small cell lung cancer (SCLC) and large cell neuroendocrine 
carcinoma (LCNEC). Expression of the Klotho gene was an important postoperative prognosticator among patients with SCLC and LCNEC $(15,16)$. Recently, the Klotho gene was shown to suppress the EMT and to inhibit transforming growth factor- $\beta 1$ (TGF- $\beta 1$ ) signaling, which is related to the invasion and metastasis of cancer (17). We hypothesized that the expression of Klotho regulates the EMT in squamous cell carcinoma. The objective of the present study was to evaluate the association between the expression of Klotho and the regulation of EMT in squamous cell lung cancers. Accordingly, we investigated how the expression of the Klotho gene regulates cell proliferation and examined its relevance to the EMT in squamous lung cancer.

\section{Materials and methods}

Patient selection. For the analysis of non-invasive squamous cell carcinoma, such as carcinoma in situ (cis), at Tokyo Medical University Hospital (Usuda J moved to Nippon Medical School on December 2012) between June 2009 and December 2011, we identified 10 patients with centrally located early lung cancers (CLELC) that had been diagnosed during a bronchoscopy performed because of abnormal sputum production and/or sputum cytological abnormalities in a mass screening. These 10 patients, who received photodynamic therapy using NPe6 (talaporfin sodium), were enrolled in the present study. This retrospective study was conducted with the approval of the Ethics Committee of Tokyo Medical University.

For the analysis of invasive squamous cell carcinoma, among 161 patients who underwent surgical resection for primary lung cancer between April 2007 and December 2008 at Nippon Medical School Hospital, 30 patients with squamous cell carcinoma were enrolled in the present study. Clinical information was extracted from the medical records. The disease stage was based on the TNM classification, 7th edition, using the International Union Against Cancer (UICC) staging system. The all-histological types were diagnosed by experienced pathologists at the Department of Pathology, Nippon Medical School Hospital, according to the histological typing of lung and pleural tumors in the WHO International Histological Classification of Tumors, 4th edition. This retrospective study was conducted with the approval of the Ethics Committee of Nippon Medical School Hospital.

Immunohistochemical staining for Klotho. We immunohistochemically examined the expression of Klotho in patients with lung squamous cell carcinoma who had undergone surgical resection or photodynamic therapy. Immunohistochemical staining for Klotho was performed on $4-\mu \mathrm{M}$ formalin-fixed, paraffin-embedded tissue sections (15-17). The slides were deparaffinized in xylene and dehydrated in a graded ethanol series. Endogenous peroxidase was blocked with $0.3 \% \mathrm{H}_{2} \mathrm{O}_{2}$ in methanol for $30 \mathrm{~min}$. All the slides were heated to $95^{\circ} \mathrm{C}$ by exposure to microwave irradiation for $20 \mathrm{~min}$. The slides were then cooled for $1 \mathrm{~h}$ at room temperature and washed in phosphate-buffered saline (PBS). Non-specific binding was blocked by pre-incubation with $1 \%$ BSA for $30 \mathrm{~min}$. After washing with PBS, the slides were incubated with an anti-Klotho antibody (KM2076; Kyowa Hakko Kirin, Tokyo,
Table I. Clinicopathologic Characteristics of the patients with centrally located early lung cancer who underwent PDT.

\begin{tabular}{ll}
\hline Characteristics & \multicolumn{1}{c}{ N0. } \\
\hline Patients & 10 \\
Age $(y)$ & $57-81(68.6)$ \\
Gender & Male: 10 \\
& Female: 0 \\
Size $(\mathrm{mm})$ & $6-20$ (10.8) \\
Endoscopic findings & Flat type: 8 \\
& Polypoid: 1 \\
CR rate & Nodular: 1 \\
Klotho expression & $100 \%$ \\
& Positive: 10 \\
\hline
\end{tabular}

Japan) at $4^{\circ} \mathrm{C}$ overnight. The slides were then incubated with anti-Klotho antibody for $30 \mathrm{~min}$ at room temperature. After washing in PBS, the slides were then incubated with a peroxidase-conjugated secondary antibody. Negative controls were prepared by omitting the primary antibody under the same experimental conditions. Antibody staining was considered positive when at least $10 \%$ of the tumor cells were stained, based on the use of a $10 \%$ cutoff level in several previous studies $(15,16)$. All the slides were examined by two observers who had no knowledge of the patients' clinical data.

Cell culture and transfection. The human squamous lung cancer cell line SQ5 and the human adenocarcinoma cell line A549 were maintained in Dulbecco's modified Eagle's medium (DMEM) supplemented with $10 \%$ fetal bovine serum (FBS). We transfected a GFP-Klotho plasmid, which was kindly provided by Dr Nabeshima (Foundation for Biomedical Research and Innovation, Kobe, Japan), into SQ5 cells using Lipofectamin 3000 transfection reagent, according to the manufacturer's protocol (Invitrogen, Carlsbad, CA, USA).

Flow cytometry. We transfected the GFP-Klotho plasmid or a GFP vector into SQ5 cells; 24 h later, the cells were pelleted by centrifugation, resuspended in PBS to a final density of $\sim 2.9 \times 10^{6}$ cells $/ \mathrm{ml}$, and then filtered through a nylon membrane to remove cell aggregates. We sorted the GFP-positive cells using flow cytometry and a FACSCanto II (BD Biosciences, CA, USA) with activation at $488 \mathrm{~nm}$ and fluorescence emission monitoring at $508 \mathrm{~nm}$ (GFP). The data acquisition and analysis were performed using FlowJo software (TreeStar, Ashland, OR, USA). A minimum of 10,000 events were collected for each analysis. We eliminated dead cells and debris from the analysis using forward-scatter and side-scatter parameters, and the remaining cells were then sorted into GFP-positive and GFP-negative populations. The resulting cells were resuspended in PBS. The protein expression levels were then analyzed using an immunoblot analysis.

Immunoblot analysis. Cells were harvested by centrifugation and washed twice with ice-cold PBS. The cell pellets were 

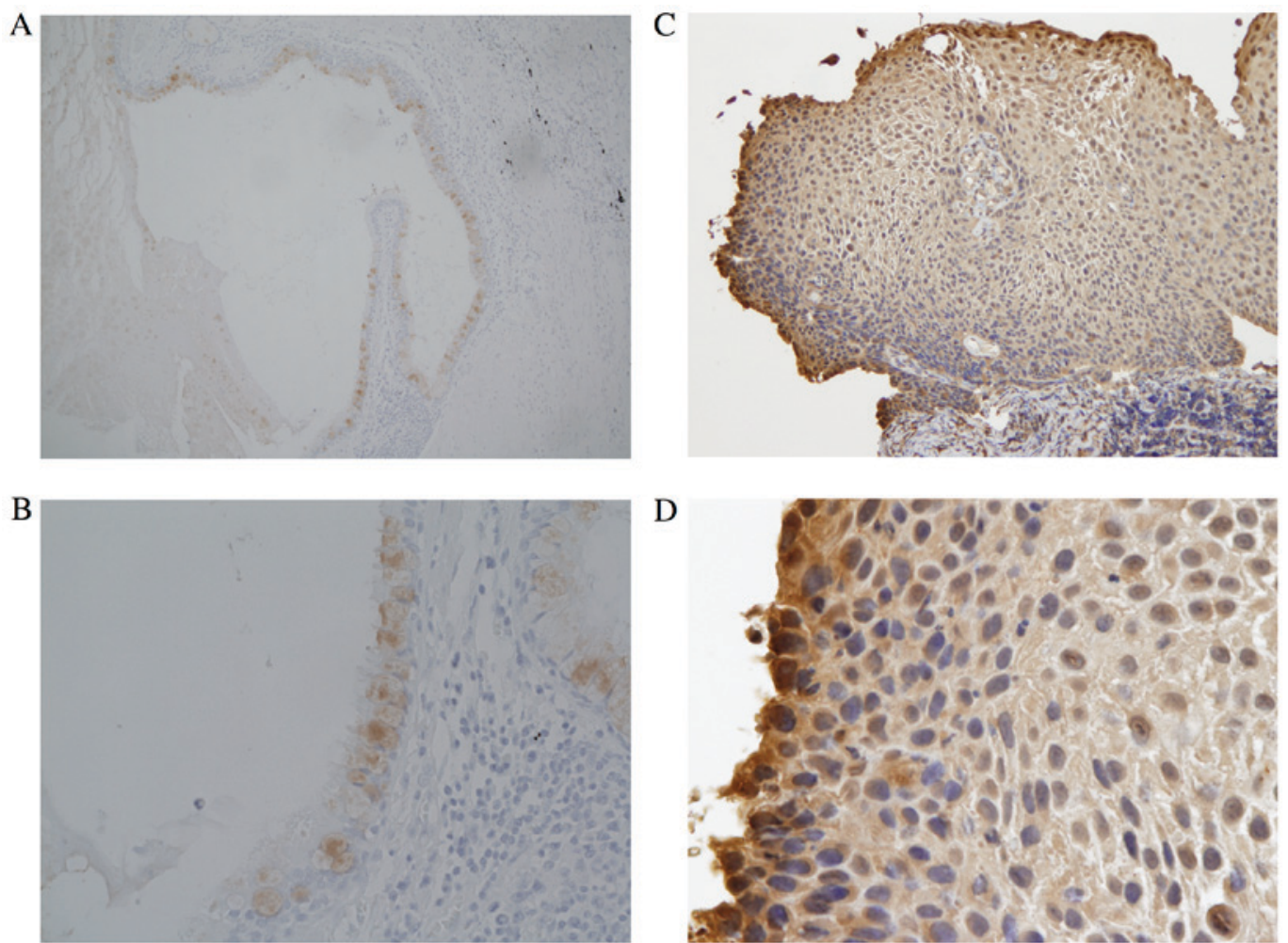

Figure 1. Immunohistochemical staining for Klotho in normal bronchial epithelial cells and centrally located early lung cancers (A, x100; B, x400). Expression of Klotho in normal epithelial cells (C, x100; D, x400). Expression of Klotho in centrally located early lung cancers.

incubated in a lysis buffer $(50 \mathrm{mM}$ Tris- $\mathrm{HCl}, \mathrm{pH} 7.6,120 \mathrm{mM}$ $\mathrm{NaCl}, 1 \%$ Triton $\mathrm{X}-100,0.2 \%$ sodium-deoxycholate and protease inhibitor). The lysates were cooled on ice for $10 \mathrm{~min}$ and then centrifuged at 14,000 x $g$ for $30 \mathrm{~min}$. Each sample of $6.56 \mu \mathrm{g}$ of total protein was separated using SDS-PAGE on $7.5 \%$ gels and transferred to polyvinylidene difluoride membranes.

The membranes, after blocking with 5\% skimmed milk, were incubated with the following antibodies: rat anti-human Klotho monoclonal antibody (1:500 dilution; Kyowa Hakko Kirin, Tokyo, Japan), rabbit anti-human E-cadherin monoclonal antibody (1:1,000 dilution), rabbit anti-human $\mathrm{N}$-cadherin monoclonal antibody (1:1,000 dilution), rabbit anti-human vimentin monoclonal antibody (1:1000 dilution), or rabbit anti-human Snail monoclonal antibody (1:500 dilution) (all from Cell Signaling Technology, Inc., Danvers, MA, USA) for $1 \mathrm{~h}$. After rinsing with PBS containing $0.1 \%(\mathrm{v} / \mathrm{v})$ Triton X-100, the membranes were incubated with goat anti-rat immunoglobulin-G ( $\operatorname{IgG})$-conjugated horseradish peroxidase (HRP) (1:10,000 dilution; Kirkegaard \& Perry Laboratories, Gaithersburg, MD, USA) for the anti-Klotho antibody or goat anti-rabbit IgG conjugated HRP (1:2,000 dilution; Cell Signaling Technology, Inc.) for the other antibodies. As a loading control, blots were probed with $\beta$-actin $(1: 1,000$ dilution; Cell Signaling Technology, Inc.). The membranes were washed and developed using western blotting enhanced chemiluminescence detection reagents (Bio-Rad Laboratories, Richmond, CA, USA).

Statistics. The statistical analyses were performed using EZR (Saitama Medical Center, Jichii Medical University, Saitama,
Japan), which is a graphical user interface for $\mathrm{R}$ (The $\mathrm{R}$ Foundation for Statistical Computing, Vienna, Austria) (18). The study variables were compared between the study groups using the Fisher's exact test for categorical variables and the Student's $t$-test for continuous variables. P-values of $<0.05$ were considered significant.

\section{Results}

Expression of Klotho in clinical specimens . To elucidate the association between cancer-invasiveness and the expression of Klotho, we first examined the expression of Klotho in lung cancer patients with squamous cell carcinoma in situ (cis) using an immunohistochemical analysis. The clinicopathological characteristics of the patients are listed in Table I. The median patient age at the time of diagnosis was 68 years (range, 57-81 years). All the patients were men and were heavy smokers with a smoking history of $>30$ pack-years. In all 10 patients with carcinoma in situ, we observed the expression of Klotho as shown in Fig. 1C and D. The expression of Klotho was observed in not only normal bronchial epithelial cells, but also centrally located early lung cancers, which were all carcinoma in situ and had been treated using PDT. The criteria for a centrally located early lung cancer (CLELC) were strictly defined in 1975 . The tumor must be located only as far as the segmental bronchi and must be a carcinoma in situ or with only limited invasion into the bronchial wall. Fig. 1C and D shows the typical features of CLELC. In these centrally located early lung cancers, cells that were located closer to the basal membrane epithelial cells had lower expression levels of Klotho. These results 
Table II. Clinicopathological characteristics of 30 patients with invasive squamous cell carcinoma who underwent lung operation.

\begin{tabular}{|c|c|}
\hline Characteristics & No. \\
\hline Total & 30 \\
\hline Age (years) & $44-82(71.7)$ \\
\hline \multicolumn{2}{|l|}{ Gender } \\
\hline Male & 25 \\
\hline Female & 3 \\
\hline \multicolumn{2}{|l|}{ Surgical procedure } \\
\hline Segmentectomy & 2 \\
\hline Lobectomy & 25 \\
\hline Pneumonectomy & 3 \\
\hline \multicolumn{2}{|l|}{ Location } \\
\hline central & 3 \\
\hline intermidiate & 10 \\
\hline peripheral & 17 \\
\hline \multicolumn{2}{|l|}{ Pathological Stage } \\
\hline IA & 10 \\
\hline IB & 4 \\
\hline IIA & 2 \\
\hline IIB & 9 \\
\hline IIIA & 5 \\
\hline Diameter (mm) & $15-90(37.3)$ \\
\hline \multicolumn{2}{|c|}{ Lymphangio-invasion } \\
\hline$(-)$ & 5 \\
\hline$(+)$ & 25 \\
\hline \multicolumn{2}{|l|}{ Lymph node meta } \\
\hline$(-)$ & 21 \\
\hline$(+)$ & 9 \\
\hline \multicolumn{2}{|l|}{ Pleural invasion } \\
\hline$(-)$ & 20 \\
\hline$(+)$ & 10 \\
\hline \multicolumn{2}{|c|}{ Pulmonary metastasis } \\
\hline$(-)$ & 29 \\
\hline$(+)$ & 1 \\
\hline \multicolumn{2}{|l|}{ Recurrence } \\
\hline Yes & 9 \\
\hline No & 21 \\
\hline \multicolumn{2}{|l|}{ Alive } \\
\hline Yes & 18 \\
\hline No & 12 \\
\hline \multicolumn{2}{|l|}{ Klotho } \\
\hline Yes & 4 \\
\hline No & 26 \\
\hline
\end{tabular}

suggest that the expression of Klotho may be associated with cancer invasiveness.

Next, to analyze invasive squamous cell carcinoma, we performed immunohistochemical staining for specimens from patients who had undergone surgical resection. The
Table III. Clinicopathologic characteristics of patients who underwent surgical resection, for Klotho positive and negative groups.

\begin{tabular}{lccc}
\hline Characteristics & Klotho (-) & Klotho (+) & P-value \\
\hline No. of patients & 26 & 4 & \\
Age (years) & 72.6 & 65.8 & 0.099 \\
Gender & & & \\
$\quad$ Male & 23 & 4 & 1 \\
$\quad$ Female & 3 & 0 & \\
$\quad$ Surgical procedure & & & \\
$\quad$ Segmentectomy & 2 & 0 & 1 \\
Lobectomy & 21 & 4 & \\
$\quad$ Pneumonectomy 3 & 0 & & \\
$\quad$ Location & & & \\
$\quad$ Central & 3 & 0 & 0.752 \\
$\quad$ Intermidiate & 8 & 2 & \\
Peripheral & 15 & 2 & \\
Pathogit Stan & & &
\end{tabular}

Pathological Stage

$\begin{array}{lccc}\text { IA } & 9 & 1 & 0.926 \\ \text { IB } & 3 & 1 & \\ \text { IIA } & 2 & 0 & \\ \text { IIB } & 8 & 1 & \\ \text { IIIA } & 4 & 1 & \\ \text { Diameter (mm) } & 37.0 & 39.2 & 0.83 \\ \text { Lymphangio-invasion } & & & \\ (-) & 4 & 1 & 0.538 \\ (+) & 22 & 3 & \end{array}$

Lymph node meta

$\begin{array}{lrr}(-) & 18 & 3 \\ (+) & 8 & 1\end{array}$

Pleural invasion

$\begin{array}{lrrr}(-) & 17 & 3 & 1 \\ (+) & 9 & 1 & \end{array}$

Pulmonary metastasis

\begin{tabular}{lrrr}
$(-)$ & 25 & 4 & 1 \\
$(+)$ & 1 & 0 & \\
Recurrence & & & \\
No & 18 & 3 & 1 \\
Yes & 8 & 1 & \\
Alive & & & \\
Yes & 16 & 2 & 1 \\
No & 10 & 2 & \\
\hline
\end{tabular}

clinicopathological characteristics of the patients with invasive squamous cell carcinoma are listed in Table II. Though the sample number in Klotho positive group is small, no significant differences in the clinicopathological characteristics were observed between the Klotho-positive group and the Klotho-negative group (Table III). However, in lung cancer patients with invasive or advanced squamous cell carcinoma, 
A

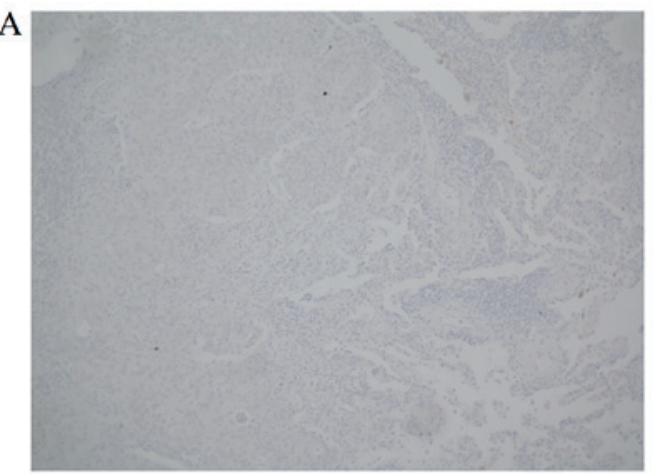

B

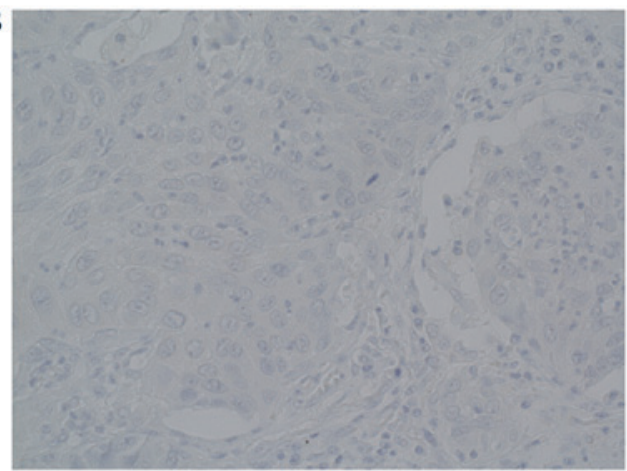

$\mathrm{C}$

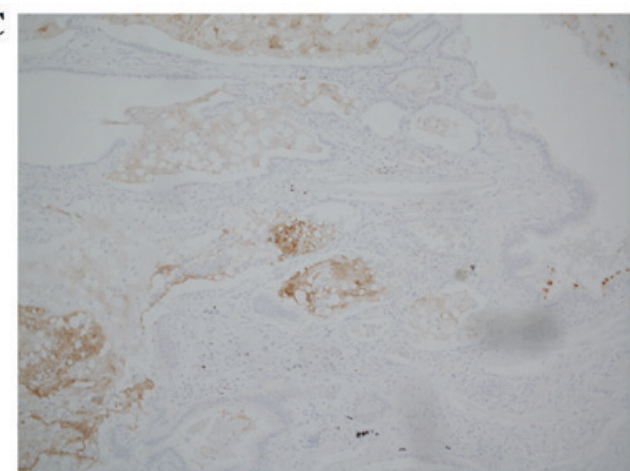

$\mathrm{D}$

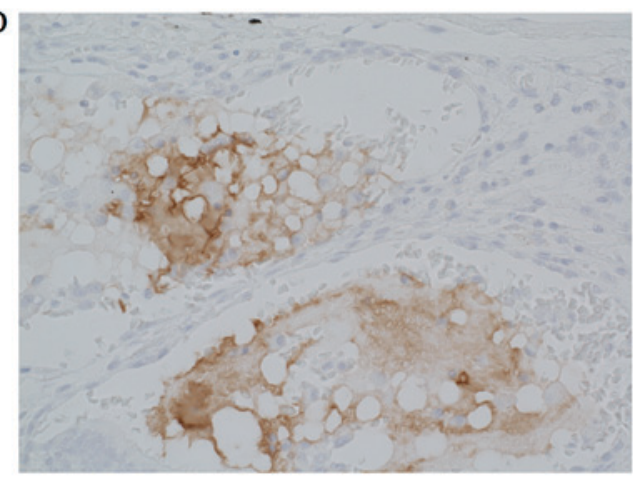

Figure 2. Immunohistochemical staining for Klotho in invasive squamous cell carcinoma (A, x100; B, x400). Loss of Klotho expression in invasive squamous cell carcinoma (C, x100; B, x400). Expression of Klotho in invasive squamous cell carcinoma.
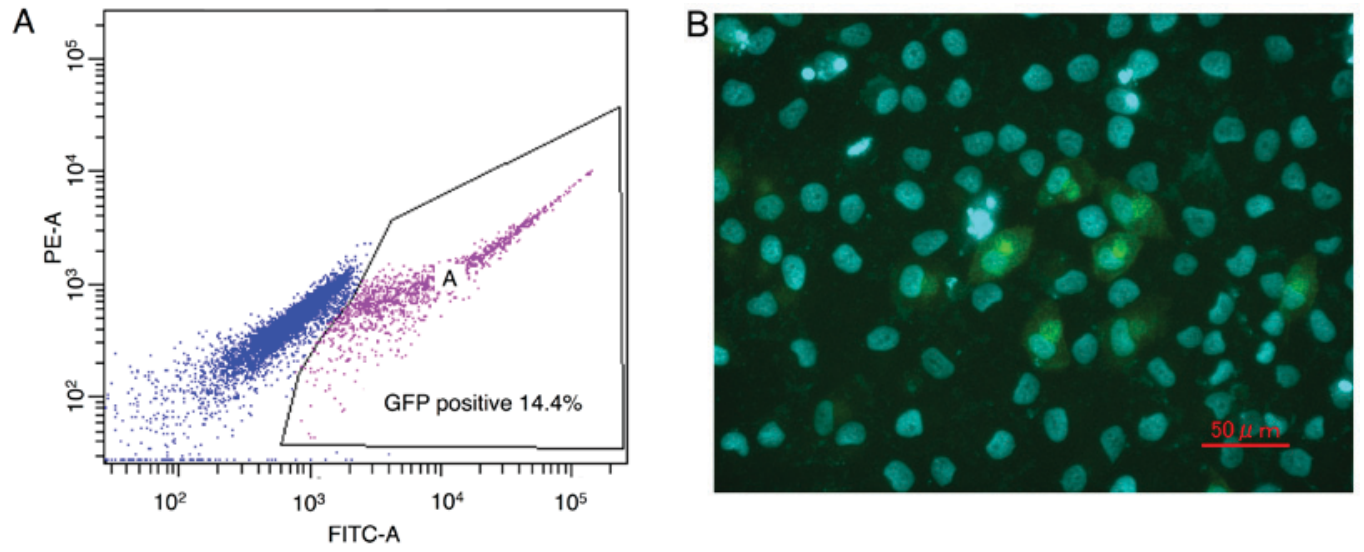

Figure 3. (A) FACS gating for the sorting of SQ5 cells with GFP. The signal from the excitation of cells with an FITC laser is plotted against the signal from the PE laser. The population with the higher FITC-A signal was selected for sorting. (B) Fluorescence cell imaging of SQ5 cells transfected with the GFP-Klotho expression vector. Several SQ5 cells transfected with the GFP-Klotho expression vector exhibited GFP in their cytoplasm.

who had undergone a complete surgical resection, Klotho expression was observed in only 4 patients (13\%) (Fig. 2). Therefore, these results suggested that the expression of Klotho may be associated with tumor development in squamous cell lung cancer.

Klotho regulated the epithelial-mesenchymal transition in vitro. We hypothesized that Klotho expression regulates EMT-related proteins, which are associated with invasion and metastasis. We next studied EMT markers such as E-cadherin, $\mathrm{N}$-cadherin, vimentin and Snail in lung squamous cancer cells. To improve the efficiency of transfection, the SQ5 cells were transfected with a GFP-Klotho expression vector and then sorted into GFP-positive or GFP-negative populations using flow cytometry. Overall, $14.8 \%$ of the cells were sorted as GFP-positive cells (Fig. 3A, B).

To determine whether Klotho expression affects the protein expressions of EMT markers, an immunoblot assay was performed (Fig. 4). The GFP-positive sorted cells showed an overexpression of Klotho. Moreover, in SQ5 cells transiently overexpressing GFP-Klotho, the expression of N-cadherin, which is a mesenchymal marker, was completely inhibited, compared with wild-type SQ5 cells or SQ5 cells transfected with only the GFP vector. Namely, the overexpression of Klotho resulted in a loss of $\mathrm{N}$-cadherin expression in the SQ5 cells. The overexpression of Klotho did not affect the regulations of other mesenchymal markers, such as vimentin or Snail, or the epithelial marker E-cadherin. Thus, the present in vitro study 


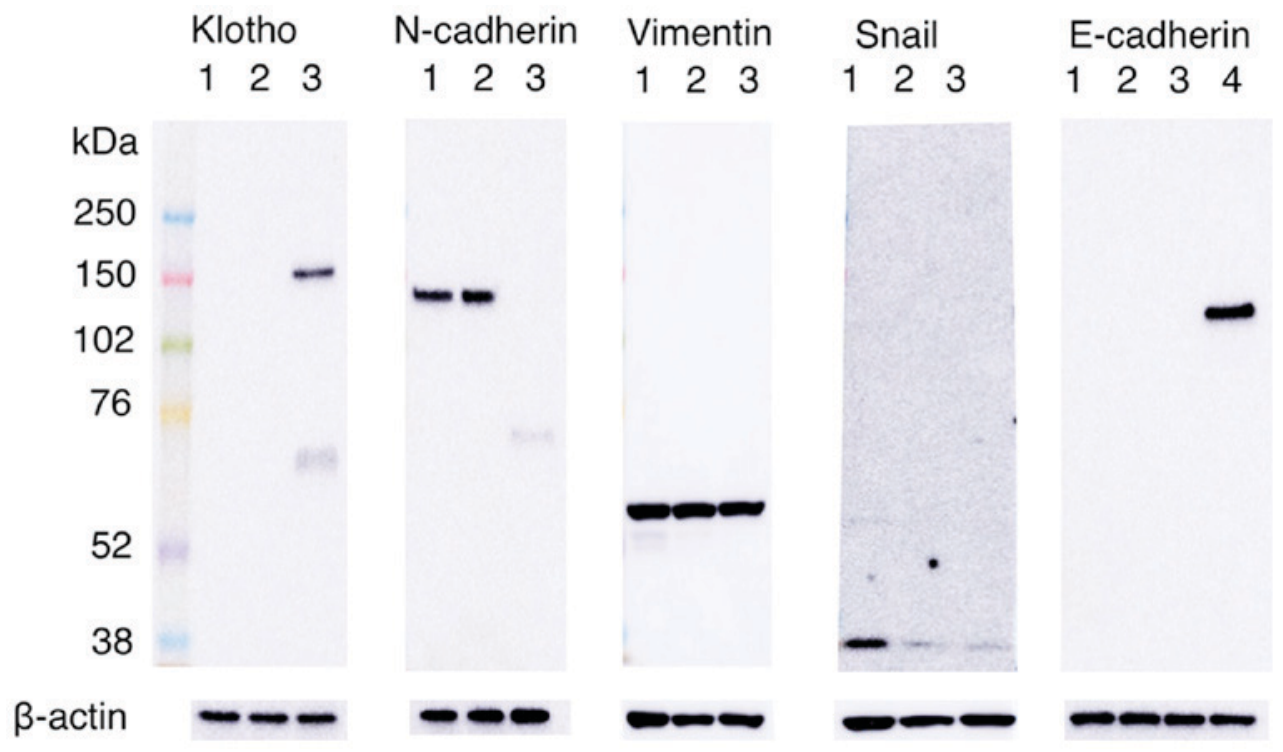

Figure 4. Immunoblot assay for SQ5 cells transfected with the GFP-Klotho expression vector. Lanes 1-3 show wild-type, GFP-vector, and GFP-Klotho transfected SQ5 cells, respectively. The overexpression of GFP-Klotho inhibited the expression of N-cadherin in SQ5 cells. In E-cadherin blot, lane 4 shows A549 cells as positive control.

confirmed that Klotho expression inhibits N-cadherin expression, which is an EMT marker, in lung squamous cancer cells.

\section{Discussion}

Several insights have emerged as a result of our immunohistochemical analyses. Klotho expression was observed not only in normal bronchial epithelial cells, but also in centrally located early lung cancers in all the patients. In patients with invasive squamous cell carcinoma, however, Klotho expression was only seen in 4 cases $(13 \%)$. Several reports have identified an association between Klotho expression and cancerous changes. Some reports have shown a reduction in the expression of Klotho in invasive breast cancers or cervical cancers, compared with normal lesions $(19,20)$. Moreover, the expression of Klotho has been observed in carcinoma in situ, but not in invasive carcinoma $(20,21)$. As shown in Figs. 1 and 2, the expression of Klotho was associated with the inhibition of cancer invasiveness, similar to the results of previous reports. These results suggest that Klotho expression is associated with invasion and that a loss of Klotho expression can promote cancer progression in squamous cell lung carcinoma.

In this study, we hypothesized that the expression of Klotho can inhibit the progression of lung cancer and regulate the EMT. To elucidate the association between the expression of Klotho and the expression of EMT markers, we examined the protein levels using a western blot analysis and a human squamous lung cancer cell line, SQ5, transfected with GFP-Klotho plasmid DNA, as shown in Fig. 4. The Klotho blot showed two bands in SQ5 cells transfected with the GFP-Klotho plasmid DNA (lane 3). This is caused by two forms: membrane Klotho and secreted Klotho. The extracellular domain is able to ectodomain shedding. Klotho protein is clipped on the cell surface by membrane-anchored proteases and the entire extra cellular domain is released into systemic circulation, functioning as a hormone $(14,17)$. We observed that the overexpression of Klotho almost completely suppressed the expression of $\mathrm{N}$-cadherin. These results indicate that Klotho inhibited the expression of $\mathrm{N}$-cadherin, which is a mesenchymal marker, and Klotho may inhibit the progression of cancer cells. The N-cadherin blot showed smaller band in GFP-Klotho transfected SQ5 cells. This appears to be caused by the protein destruction of $\mathrm{N}$-cadherin or the suppression of the $\mathrm{N}$-cadherin expression at the mRNA level, followed by overexpression of Klotho. The E-cadherin blot did not show any band in three SQ5 samples. This result indicates that E-cadherin is not expressed originally and E-cadherin expression is not caused by Klotho transfection in SQ5 cells. The Snail blot showed that both the GFP-vector control and the GFP-Klotho samples have a reduced level of Snail. This may suggest that the transfection may be causing this reduction.

The regulation of $\mathrm{N}$-cadherin expression by Klotho is reportedly related to several signaling pathways. The Wnt signaling pathway inhibits glycogen synthase kinase- $3 \beta$, which stabilizes $\beta$ catenin, promoting $\mathrm{N}$-cadherin expression. Klotho can inhibit the activation of the Wnt signaling pathway (22). Lee reported that Klotho functioned as a Wnt antagonist in a cervical cancer cell line and that the expression of Klotho in SiHa cells resulted in a decrease in N-cadherin (23). In addition, the IGF-1 signaling pathway has been shown to have a strong influence on the EMT process (14). Klotho binds to a cell-surface receptor to inhibit the activation of insulin and IGF-1 and repress the intercellular signals of insulin and IGF-1. Moreover, Klotho has been reported to inhibit TGF- $\beta 1$-induced EMT responses, resulting in an increase in $\mathrm{N}$-cadherin and a decrease in E-cadherin in A549 cells (17). From these studies, we can speculate that Klotho may play a critical role as a suppressor of the expression of $\mathrm{N}$-cadherin, thereby inhibiting the Wnt, IGF-1, and TGF- $\beta 1$ signaling pathways simultaneously.

To our knowledge, this is the first study that the Klotho gene was a suppressor of the EMT in lung squamous cell carcinoma. However, the limitations of this study include that we used only SQ5 cell line, that we did not clarified whether the 
overexpression of Klotho can inhibit the invasiveness of cancer cells, and that we did not evaluate expression of E-cadherin markers in immunohistochemical analysis. Therefore, additional studies are needed to make a more definitive conclusion regarding the ability of Klotho to inhibit cellular infiltration, cell proliferation, and cell motility in more squamous lung cancer cell lines. We would like to investigate expression of EMT markers in immunohistochemical analysis in the future.

In this immunohistochemical analysis of specimens from patients with lung squamous cell carcinoma who had undergone surgical resection, Klotho expression did not have a significantly favorable effect on patient outcome (data not shown). There was, however, a trend for an improvement in disease-free survival consistent with Klotho expression. We previously reported that the expression of Klotho was an important prognosticator for lung large cell neuroendocrine carcinoma and lung small cell carcinoma $(15,16)$. Further studies in more patients with lung squamous cell carcinoma are needed.

Because the EMT has been shown to affect not only carcinoma invasion or metastasis but also the acquisition of cancer-stem cell traits or resistance to anticancer drugs or radiation therapy, the EMT may play an important role as a treatment target for cancer. Therefore, we suggest that Klotho may regulate the EMT and could be useful as a new and effective anti-cancer drug in lung squamous cell carcinoma. In the future, we hope to develop innovative treatment strategies for squamous cell lung carcinoma that include not only postoperative adjuvant chemotherapy, but also new drug discovery.

\section{Acknowledgements}

This study was supported in part by Grant-in-Aid for Scientific Research (C) from Japan Society for the Promotion of Science (JSPS), KAKENHI 16K10693 (J.U.) and supported by the Research on Development of New Medical Devices from Japan Agency for Medical Research and development (AMED), 16hk0102025h0002 (J.U.). We would like to thank Professor Yo-ichi Nabeshima, Laboratory of Molecular Life Science, Institute of Biomedical Research and Innovation Foudation for Biomedical Research and Innovation, for giving Klotho-GFP plasmid DNA.

\section{References}

1. American Cancer Society: Cancer Facts \& Figures 2016. American Cancer Society, Atlanta, 2016.

2. International Agency for Research on Cancer: GLOBOCAN 2012: Estimated Cancer Incidence, Mortality and Prevalence Worldwide in 2012. http://globocan.iarc.fr/Default.aspx. Accessed on August 22, 2016.

3. Maemondo M, Inoue A, Kobayashi K, Sugawara S, Oizumi S, Isobe H, Gemma A, Harada M, Yoshizawa H, Kinoshita I, et al: Gefitinib or chemotherapy for non-small-cell lung cancer with mutated EGFR. N Engl J Med 362: 2380-2388, 2010.

4. Mitsudomi T, Morita S, Yatabe Y, Negoro S, Okamoto I, Tsurutani J, Seto T, Satouchi M, Tada H, Hirashima T, et al: Gefitinib versus cisplatin plus docetaxel in patients with non-small-cell lung cancer harbouring mutations of the epidermal growth factor receptor (WJTOG3405): An open label, randomised phase 3 trial. Lancet Oncol 11: 121-128, 2010.

5. Shaw AT, Kim DW, Nakagawa K, Seto T, Crinó L, Ahn MJ, De Pas T, Besse B, Solomon BJ, Blackhall F, et al: Crizotinib versus chemotherapy in advanced ALK-positive lung cancer. N Engl J Med 368: 2385-2394, 2013.
6. Soria JC, Felip E, Cobo M, Lu S, Syrigos K, Lee KH, Göker E, Georgoulias V, Li W, Isla D, et al: Afatinib versus erlotinib as second-line treatment of patients with advanced squamous cell carcinoma of the lung (LUX-Lung 8): An open-label randomised controlled phase 3 trial. Lancet Oncol 16: 897-907, 2015.

7. Rizvi NA, Mazières J, Planchard D, Stinchcombe TE, Dy GK Antonia SJ, Horn L, Lena H, Minenza E, Mennecier B, et al: Activity and safety of nivolumab, an anti-PD-1 immune checkpoint inhibitor, for patients with advanced, refractory squamous non-small-cell lung cancer (CheckMate 063): A phase 2, single-arm trial. Lancet Oncol 16: 257-265, 2015.

8. Thiery JP: Epithelial-mesenchymal transitions in tumour progression. Nat Rev Cancer 2: 442-454, 2002.

9. Singh A, Greninger P, Rhodes D, Koopman L, Violette S, Bardeesy N and Settleman J: A gene expression signature associated with 'K-Ras addiction' reveals regulators of EMT and tumor cell survival. Cancer Cell 15: 489-500, 2009.

10. Mani SA, Guo W, Liao M-J, Eaton EN, Ayyanan A, Zhou AY, Brooks M, Reinhard F, Zhang CC, Shipitsin M, et al: The epithelial-mesenchymal transition generates cells with properties of stem cells. Cell 133: 704-715, 2008.

11. Wellner U, Schubert J, Burk UC, Schmalhofer O, Zhu F, Sonntag A, Waldvogel B, Vannier C, Darling D, zur Hausen A, et al: The EMT-activator ZEB1 promotes tumorigenicity by repressing stemness-inhibiting microRNAs. Nat Cell Biol 11: 1487-1495, 2009.

12. Kuro-o M, Matsumura Y, Aizawa H, Kawaguchi H, Suga T, Utsugi T, Ohyama Y, Kurabayashi M, Kaname T, Kume E, et al: Mutation of the mouse klotho gene leads to a syndrome resembling aging. Nature 390: 45-51, 1997.

13. Matsumura Y, Aizawa H, Shiraki-lida T, Nagai R, Kuro-o M and Nabeshima Y: Identification of the human klotho gene and its two transcripts encoding membrane and secreted Klotho protein. Biochem Biophys Res Commun 242: 626-630, 1998.

14. Kurosu H, Yamamoto M, Clark JD, Pastor JV, Nandi A, Gurnani P, McGuinness OP, Chikuda H, Yamaguchi M, Kawaguchi $\mathrm{H}$, et al: Suppression of aging in mice by the hormone Klotho. Science 309: 1829-1833, 2005.

15. Usuda J, Ichinose S, Ishizumi T, Ohtani K, Inoue T, Saji H, Kakihana M, Kajiwara N, Uchida O, Nomura M, et al: Klotho predicts good clinical outcome in patients with limited-disease small cell lung cancer who received surgery. Lung Cancer 74 : 332-337, 2011.

16. Usuda J, Ichinose S, Ishizumi T, Ohtani K, Inoue T, Saji H, Kakihana M, Kajiwara N, Uchida O, Nomura M, et al: Klotho is a novel biomarker for good survival in resected large cell neuroendocrine carcinoma of the lung. Lung Cancer 72: 355-359, 2011

17. Doi S, Zou Y, Togao O, Pastor JV, John GB, Wang L, Shiizaki K, Gotschall R, Schiavi S, Yorioka N, et al: Klotho inhibits transforming growth factor-betal (TGF-beta1) signaling and suppresses renal fibrosis and cancer metastasis in mice. J Biol Chem 286: 8655-8665, 2011.

18. Kanda Y: Investigation of the freely available easy-to-use software 'EZR' for medical statistics. Bone Marrow Transplant 48: 452-458, 2013

19. Aviel-Ronen S, Rubinek T, Zadok O, Vituri A, Avivi C, Wolf I and Barshack I: Klotho expression in cervical cancer: Differential expression in adenocarcinoma and squamous cell carcinoma. J Clin Pathol 69: 53-57, 2016.

20. Wolf I, Levanon-Cohen S, Bose S, Ligumsky H, Sredni B, Kanety H, Kuro-o M, Karlan B, Kaufman B, Koeffler HP and Rubinek T: Klotho: A tumor suppressor and a modulator of the IGF-1 and FGF pathways in human breast cancer. Oncogene 27: 7094-7105, 2008

21. Chang B, Kim J, Jeong D, Jeong Y, Jeon S, Jung SI, Yang Y, Kim KI, Lim JS, Kim C and Lee MS: Klotho inhibits the capacity of cell migration and invasion in cervical cancer. Oncol Rep 28: 1022-1028, 2012.

22. Chen B, Ma X, Liu S, Zhao W and Wu J: Inhibition of lung cancer cells growth, motility and induction of apoptosis by Klotho, a novel secreted Wnt antagonist, in a dose-dependent manner. Cancer Biol Ther 13: 1221-1228, 2012

23. Lee J, Jeong DJ, Kim J, Lee S, Park JH, Chang B, Jung SI, Yi L, Han Y, Yang Y, et al: The anti-aging gene KLOTHO is a novel target for epigenetic silencing in human cervical carcinoma. Mol Cancer 9: 109, 2010. 